\title{
Free Revascularized Fibula Graft for Reconstruction of Mandibular Continuity Defects
}

\author{
Q B Rahman ${ }^{1}$, R Karmakar ${ }^{2}$, Md. Aftabuddin ${ }^{3}$
}

\begin{abstract}
:
Mandibular reconstruction represents a challenge to the oral and maxillofacial surgeon and has been revolutionized by the modern microvascular techniques Objective: To evaluate the usefulness of the free vascularized fibula graft for reconstruction of discontinuity defects of Mandible after resections of benign aggressive odontogenic tumours. The results of 7 vascularized free fibula graft without a skin paddle that were used for mandibular reconstruction is presented. The "double barrel" technique was used in 1 case. The donor site was closed directly in all cases. All procedures were performed by the same surgical team in oral and Maxillofacial Surgery department of BSMMU and Health \& Hope Hospital from January, 2006 to December 2011. A total of patients (3 males, 4 females) were treated. All flaps except 1 were viable. Success rate was $85.71 \%$. The average length of the fibula graft was $10.96 \mathrm{~cm}$, and the number of osteotomies ranged from 0 to 3. There were no long-term functional complications in the lower leg. The fibula flap provides a successful bone graft for mandibular restoration with an acceptably low complication rate.
\end{abstract}

Key words: mandibular reconstruction; free fibular graft; microvascular anastomosis.

\section{Introduction:}

The mandible constitutes a significant part of the facial skeleton, forming the profile and contour of the lower third of the face. It is the structural foundation for the lower dentition as well as the origin for the tongue muscles. The muscles of mastication are also inserted into this bone. The mandible is vital for crucial functions like speech, chewing, swallowing, oral competence as well as the facial appearance In cases of reconstruction of a discontinuity defect of the

1. Dr. Quazi Billur Rahman, Associate Professor, Department of Oral and Maxillofacial Surgery, Bangabandhu Sheikh Mujib Medical University (BSMMU), Dhaka,

2. Dr. Rajan Karmakar, Oral and Maxillofacial Surgeon, BSMMU, Dhaka.

3. Dr. Md. Aftabuddin, Professor and Chairman, Department of Cardiac Surgery, Bangabandhu Sheikh Mujib Medical University (BSMMU).

\section{Corresponding Author:}

Dr. Quazi Billur Rahman, Associate Professor, Department of Oral and Maxillofacial Surgery, Bangabandhu Sheikh Mujib Medical University (BSMMU), Dhaka. mandible the surgeon has a major responsibility to maximize function as well as cosmesis and to preserve quality of life, restoring mastication, speech, and appearance. Because of the great importance and the different functions of the mandible, its reconstruction is a major challenge and is a subject of continuous study for the surgeon. ${ }^{1}$

A number of donor sites have been proposed for oral cavity and bone replacement. ${ }^{2-7}$ However, at this time; the microvascularized free fibula flap (VFFF) represents the state-of-the-art technique for reconstruction of continuity defects of mandible.

Vascularized bone is historically reserved for more difficult secondary reconstructions where a large defect existed, where soft tissue is inadequate, or where the recipient bed has been compromised by radiation, chronic infection, or previous surgery. Even in those adverse situations the success rate has been reported to be very high. Later, this method was also successfully applied in cases of primary reconstruction in aggressive odontogenic tumors. ${ }^{8,9}$

Taylor et al. ${ }^{10}$ were the first to report the transfer of the free fibula flap. In 1989, the method was adapted to the restoration of segmental mandibulectomy defects by Hidalgo. ${ }^{7}$ Since that time, the use of this flap has gained in popularity.

The free fibular flap has been reported to have many advantages over the various other sources of vascularized bone that have been used for mandibular reconstruction. In comparison with other free flaps, its ability to provide the largest bone length that allows reconstructing even after complete jaw resections. ${ }^{11,12}$ with good bone quality and reasonable quantity \& consistent anatomy and it is more suitable for accepting dental implants. ${ }^{13-16}$ that serve for functional reconstruction. It allows for combination with an extensive, relatively thin, skin island for reconstruction of mucosa or skin defects. ${ }^{13,14,17}$

The blood supply to the fibula is dual \& provided by the periosteum which is segmental type and by an endosteal vessel which directly enters the bone. The vascular pedicle is of adequate diameter and of sufficient length (about 6-10 $\mathrm{cm}$ ), which avoids the need for a vein graft and improves the rate of success. ${ }^{18,19}$ The path of the pedicle is intramuscular and accompanies the bone parallel to it at a distance of about $1 \mathrm{~cm}$. These characteristics permit the execution of osteotomies when necessary without compromising bone viability, even when they are separated a little more than 1 $\mathrm{cm}$ from one another, as long as the periosteum is not removed $^{20}$.The donor site morbidity is low, and its location facilitates a 2-team approach. ${ }^{12-14,17-21}$

The aim of this article is to report our clinical experience with the use of the vascularized free fibula flap without a skin paddle to restore a mandibular defects following surgical ablation. 


\section{Materials and Methods:}

The study group consisted of patients treated for reconstruction of continuity defects of the mandible, using the fibular vascularized free flap, between 2006--2011.All procedures were performed in BSMMU and Health \& Hope Hospital, Dhaka and by the same surgical team. Hospital charts were reviewed retrospectively for patients' age, gender, location and extension of the defect. The surgery time, flap ischemia time, and perioperative details were recorded. The length of the fibula, the number of osteotomies and the recipient vessels used for anastomosis were also recorded. No interpositional vein grafts were required. In addition, donor and recipient site complications were also recorded and analyzed.

\section{Surgical technique:}

Patients were evaluated clinically, specifically for dorsalis pedis and tibialis posterior pulses. Doppler study was done preoperatively angiography was not performed. Consent was taken before surgery. The patient was placed in supine position with the hip and knee in flexion and internal rotation. The head of the fibula and lateral malleolus were marked and with a straight line connecting the two, the common peroneal nerve was usually $2 \mathrm{~cm}$ below fibula head. The standard lateral approach was used and dissection was performed without a tourniquet. The skin incision was commenced $6 \mathrm{~cm}$ below the fibula head and over the previously marked line. The incision was deepened through the skin, subcutaneous tissue and the fascia overlying the peroneus muscle. Then the peroneus muscle was retracted anteriorly and lateral aspect of fibula was approached through the intermuscular septum between the lateral and posterior compartments. The peroneal muscles were elevated supraperiosteally to the fibula and reflected anteriorly. The thin anterior crural septum was incised to allow dissection in the anterior compartment and anterior tibial vessel was protected. Dissection then proceeds anteromedially until the interosseus septum was reached. The interosseus septum was divided over its full lenth and fibula was divided $6 \mathrm{~cm}$ below the fibula head and $6 \mathrm{~cm}$ above the lateral malleolus. Removal of $1 \mathrm{~cm}$ segment of bone at lower end was done to visualize the pedicle which was located under the tibialis posterior muscle. Further dissection was facilitated by traction and rotation of the fibula with bone clamps/ holder. The dissection was carried out until the bifurcation of peroneal tibial trunk. Only the peroneal vessels and a cuff of flexor halluces longus muscle were preserved in the harvested fibula .Vessel preparation was done before vessel divide. Then the flap was divided and osteotomies were performed opposite from the peroneal vessels and kept to a minimum in order to preserve periosteal vascular supply. The contoured mandible was next inserted into the defect and attached to the remaining mandibular segments by fixation with titanium miniplate. Recipient vessel was prepared and revascularized the fibula graft by anastomised to the recipient site vessels with standard microvascular technique. Return of circulation to the graft was demonstrated by periosteal bone bleeding. The donor site and recipient site was closed with usual technique and the lower leg was immobilized with a posterior splint from 5 days to a week.
Then patient mobilize with partial weight bearing. Ankle and foot movements were encouraged.

\section{Results:}

There were 7 patients, 3 males and 4 females. The age ranged from 18 to 45 years with average age of 33.57 years. The location of the lesions at body \& symphysis and type of defect central (symphysis), lateral (body) and combination of both. All cases were primarily reconstructed. Results are presented on table I. Duration of surgery was 8 hour to 12 hour (average $7 \mathrm{~h} 15 \mathrm{~min}$ ). Average length of fibula was 10 $\mathrm{cm}$ ranging from $8-12 \mathrm{~cm}$ which is presented on Table II. The fibula was osteotomized maximum up to 3 pieces. No skin island was harvested. Bony segments were fixed by miniplates in all cases. All donor sites were closed by primary closure in all cases. The vessels used for anastomosis were facial artery and vein. All vessels were anastomosed by end to end technique. Partial double barrel technique was used in 1 case. All the flaps and except 1 were successful, success rate being $85.71 \%$.No significant post operative complication was observed in recipient sites and donor site.

Table:I Patient Data

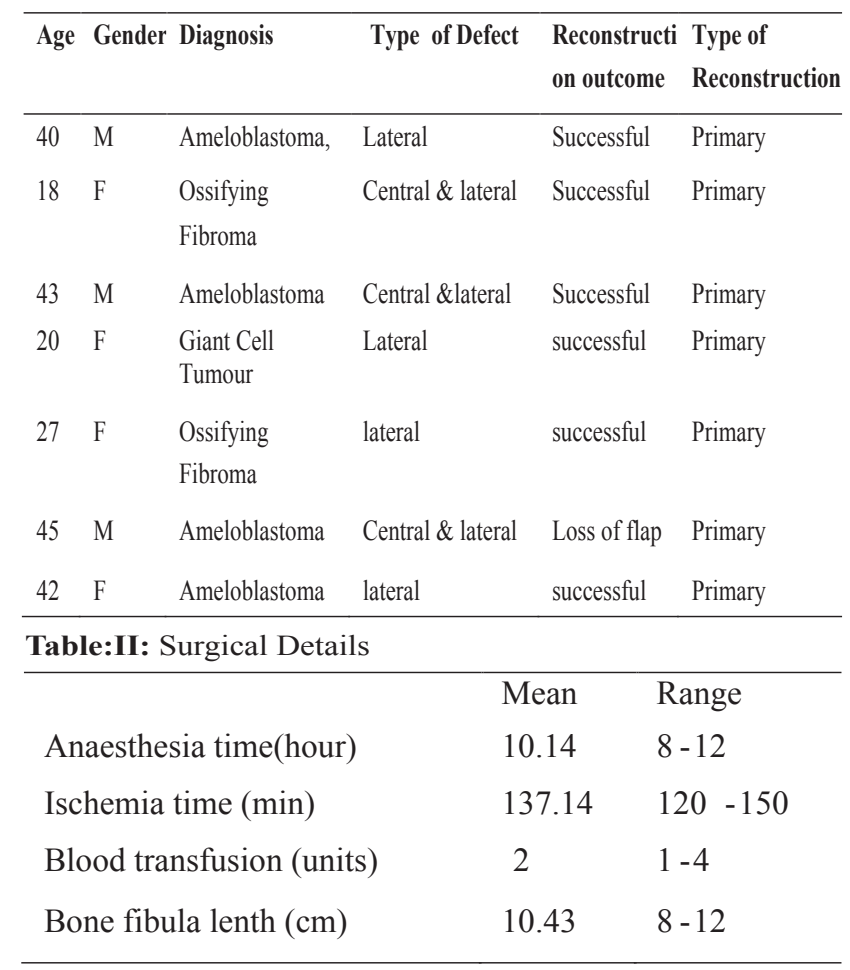

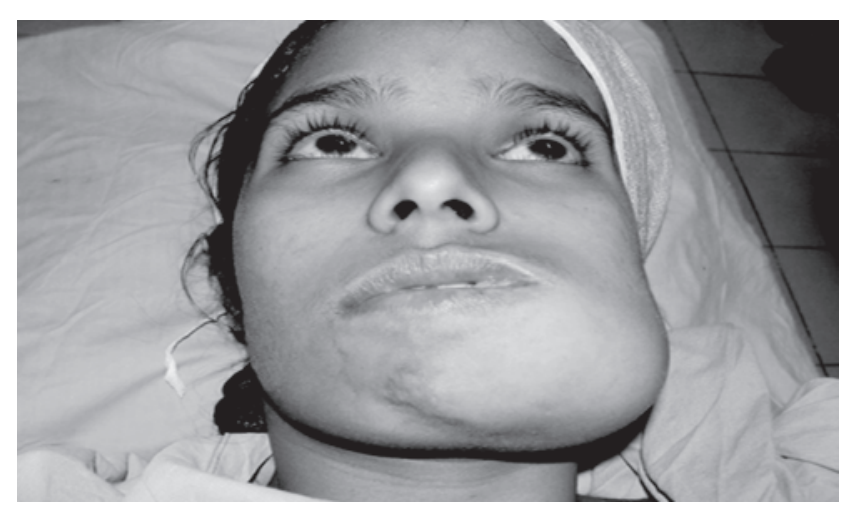

Fig: 1 Preoperative picture showing left mandiblular growth 


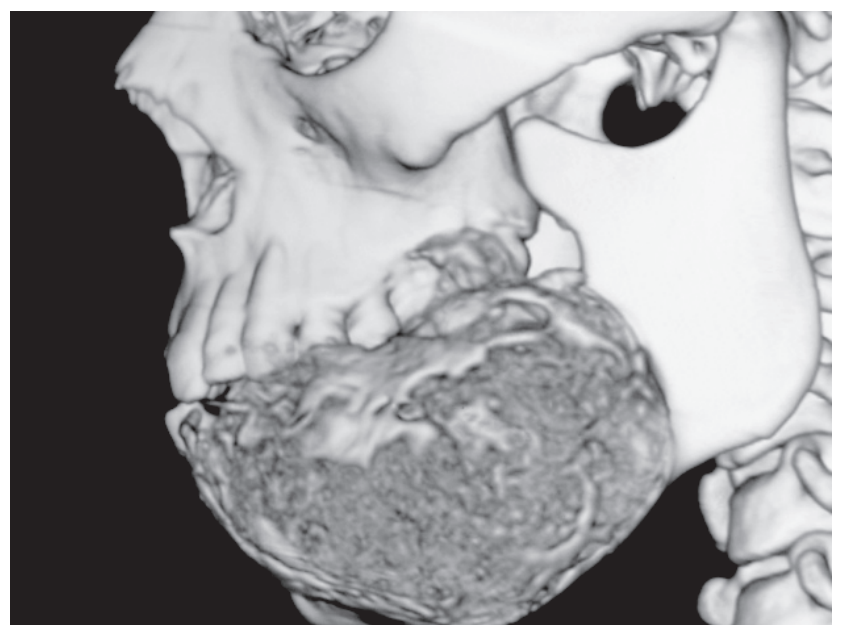

Fig:2.3-D CT-scan showing extension of tumour

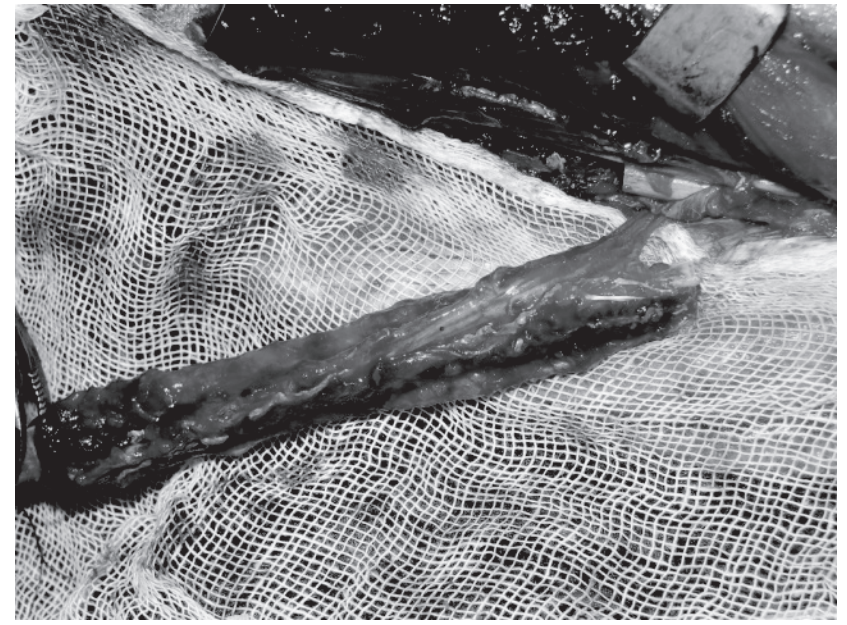

Fig:3 Harvesting Fibula Graft connected with Pedicle

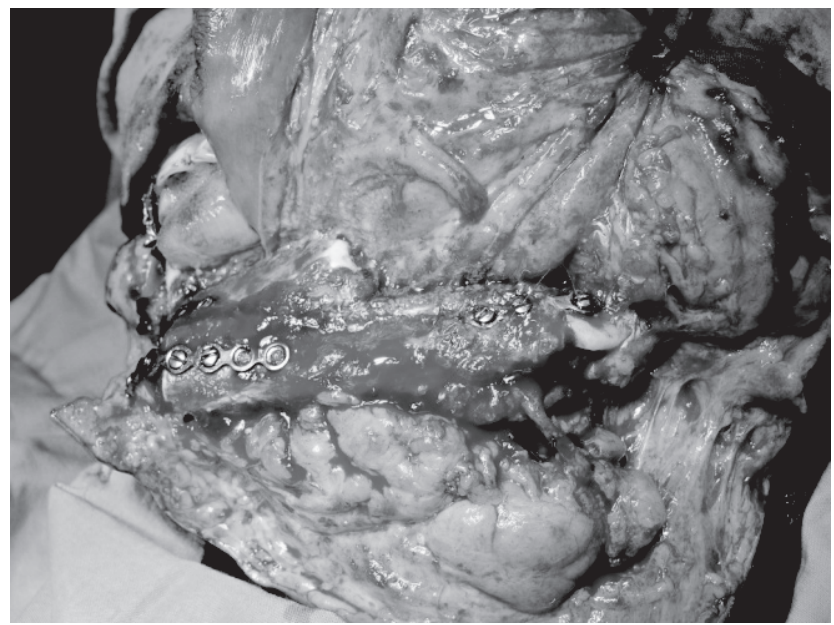

Fig: 5 Reconstruction of mandible with partial double barrel technique with complete of anastomosis

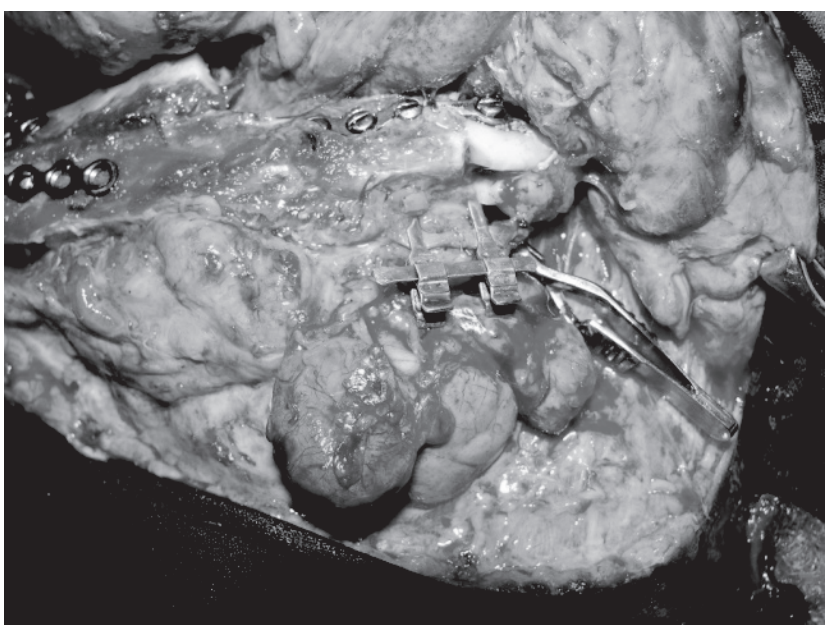

Fig:4 Peroperati ve view of anastomosis

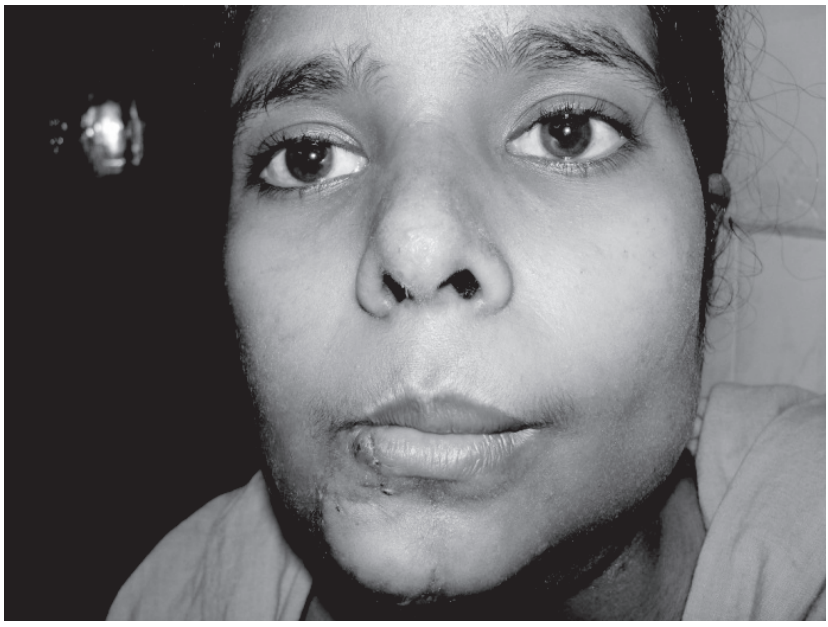

Fig:6 Facial appearance 1month after surgery

\section{Discussion:}

Malignant tumors and aggressive odontogenic tumors are the most frequent causes of mandibular defects. Bone, teeth, and soft tissue can be resected for oncologic purposes, with a significant decrease in quality of life. Nowadays, reconstruction of defects generated after tumor ablation is mandatory to obtain satisfactory results, and control of disease is not the unique aspect to be considered in an adequate complete treatment. The main goal of mandible reconstruction is early restoration of form, function and aesthetics of the face. In the past the limitations of nonvascularized bone grafts and alloplastic materials mitigated against satisfactory functional reconstruction. ${ }^{22}$ Various non-vascular bone grafts, alloplastic materials used alone or in combination with pedicle soft tissue flaps show a high rate of failure due to infection, extrusion, resorption and poor recipient vascular bed. ${ }^{23,24}$ Advances in the micro vascular surgery have allowed the transfer of well vascular autologous tissues from the distant sites like fibula, iliac crest, rib, scapula etc to achieve functional and aesthetic rehabilitation. ${ }^{25}$ Among the free flap donor sites used for mandibular reconstruction, the fibula is becoming a very popular choice. It provides a rigid, strong, tubular shaped, and has enough cortical bone stock with up to $25 \mathrm{~cm}$ of bone length and can maintain a consistent shape throughout its 
mandible defects. ${ }^{7,26}$ Its blood supply courses in parallel along the length, guaranteeing adequate vascularity to the osteotomized segments. ${ }^{27}$ For any flaps, the complications are divided into the recipient and donor site. The intraoperative complications on both sites are unremarkable. Immediate post operative complications are best avoided by a careful patient history, experienced surgeon hands and proper medications like anticoagulants, antibiotics, analgesics and anti-inflammatory agents. ${ }^{28}$ In every case, during the anastomosis of vessels, copious amount of irrigation mixed with low concentration of heparin was used. The recommended concentration of heparin used is 10 to 100 $\mathrm{U} / \mathrm{ml}$, which removes the debris on the vessels and maintains the patency of the vessels. ${ }^{28}$ There were no long term disabilities related to donor site in this study, as reported by different authors. ${ }^{2}, 18,29-32$ In this study, out of 7 cases only in one case there was complete graft failure which was reconstructed with reconstruction plate, the success rate was $85.15 \%$ in terms of the bony tissue transplant. Hidalgo 7 reported $100 \%$ success in bony transplant. Cordeiro et al. ${ }^{18}$ also reported $100 \%$ success of free flap. Abdal 30 success rate was $81 \%$; 3 free flap failures due to venous thrombosis. Hidalgo et al. ${ }^{14}$ success rate was $98 \%$. Even though the success rate of this study is $85.15 \%$, aesthetic and functional outcomes are not outstanding, which could be further improved by a combination of other soft tissue flaps, placements of implants, encouraging the patient towards the distraction osteogenesis of transplanted fibula and use of artificial prosthesis. One of the main disadvantages of the flap is the height of the bone, which is lower than the iliac crest. It could be considered a problem mainly in edentulous patients, in which non-atrophied mandibles should be encountered. However by means of the use of the partial double-barrel fibular graft, this condition can be attenuated and enough mandibular height is obtained which was done in 1 case, The use of the double-barrel free vascularized fibula flap was first described by Bahr et al. ${ }^{16}$ in 1998.this problem can also be addressed by vertical distraction osteogenesis of transplanted fibula or onlay bone grafting over the fibula 6-12 months after transplantation.

\section{Conclusion:}

Microvascular reconstruction is currently the best method of mandibular reconstruction, enjoying very high success rates with good to excellent aesthetic and functional results in the majority of patients .According to the literature and our limited experience, the fibula flap is a safe and reliable option for mandible reconstruction. However, further study could be done with larger sample size and greater logistic support.

\section{References:}

1. Takushima A, Harii K, Assato H, et al. Mandibular reconstruction using microvascular free flaps: a surgical analysis of 178 cases. Plast Reconstr Surg 2001;108(6):1555-63.

2. Serafin D, Villareal-Rios A, Georgiade NG: A rib-

3. $1977 ; 30: 263$. containing free flap to reconstruct mandibular

Bell MSG, Barron PT: A new method of oral reconstruction using a free composite foot flap. Ann
4. Taylor GI: Reconstruction of the mandible with free composite iliac bone grafts. Ann Plast Surg 1982;9:361.

5. Soutar DS, Scheker LR, Tanner NSB, et al: The radial forearm flap: A versatile method for

6. intraoral reconstruction. $\mathrm{Br} \mathrm{J}$ Plast Surg 1983;36:1.

Schwartz WM, Banis J, Newton E, et al: The osteocutaneous scapular flap for mandibular and

7. maxillary reconstruction. Plast Reconstr Surg 1986;77:530.

Hidalgo DA. Fibula free flap:a new method of mandible

8. reconstruction. Plastic and Reconstructive Surgery Jul 1989; 84(1): 71-9.

Haughey BH, Fredrickson JM, Lerrick AJ, et al: Fibular

9. and iliac crest osteomuscular free flap reconstruction of the oral cavity. Laryngoscope 1994; 104:1305.

10. Disa JJ, Cordeiro PG: Mandible reconstruction with microvascular surgery. Semin Surg Oncol

11. 2000;19:226.

Taylor GI, Miller GDH, Hamm FJ: The free vascularized bone graft. Plast Reconstr Surg 1975;55:533.

Leon BR, Carrillo FJ, Gonzalez HM, et al: Mandibular

12. reconstruction with the free vascularized fibular flap: Utility of three dimensional computerized tomography. $\quad \mathrm{R}$ e c o $\mathrm{n} \mathrm{s} \mathrm{t} \mathrm{r}$ Microsurg 1999;15:91.

13. Santamaria E, Wei FC, Chen HC: Fibula osteoseptocutaneous flap for reconstruction of

14. Reconstr Surg 1988;101:921.

Disa JJ, Winters RM, Hidalgo DA: Long-term evaluation

15. of bone mass in free fibula flap mandible reconstruction. Am J Surg 1997; 174:503

Hidalgo DA, Rekow A. A series of 60 consecutive 6. fibular free flap mandible reconstructions. Plast Reconstr Surg 1995; 96: 585-96.

Serra JM, Paloma V, Mesa F, et al: The vascularized

17. fibula graft in mandibular reconstruction. J 1991;49:244.

8. Bahr W, Stoll P, Wachtet R. Use of the "double barrel"

18. free vascularized fibula in mandibular reconstruction. J Oral Maxillofac Surg 1998;56:38-44.

Lee JH, Kim MJ, Kim JW: Mandibular reconstruction $\begin{array}{ccc}\text { with free } & \text { vascularized fibular flap. J } \\ \text { Craniomaxillofac } & \text { Surg 1995;23:20. } & \end{array}$

Cordeiro PG, Disa JJ, Hidalgo DA, et al. Reconstruction

20. of the mandible with osseous free flaps: a 10-year $\begin{array}{ll}\text { experience with } 150 \text { consecutive patients. Plast } \\ \text { Reconstr } & \text { Surg 1999; 104(5):1314-20. }\end{array}$

21. Kroll SS, Schsterman MA, Reece GP, et al. Choice of flap and incidence of free flap success.

22. Plast Reconstr Surg 1996; 98(3):459-63

Hidalgo DA. Aesthetic improvements in free-flap mandible reconstruction. Plast Reconstr Surg 
23. Peter WB, Stephen AS, Jarg-Erich H. Maxillofacial Publication Surgery. London:Churchill Living Stone

24. 527-528,549-556.

Fonceka RJ. Oral and Maxillofacial Surgery.

25. Philadelphia:Sounders Company 2000:364-365,407-12.

Ritvik PM, Daniel GD. Mandibular reconstruction in 2004: an analysis of different techniques. Current

26. Opinion in Otolaryngology \& Head and Neck Surgery 2004; $12: 288-93$

Wolff KD, Hözle F. Raising of Microvascular Flaps-A

27. Approach. Berlin:Springer-Verlag Berlin Heidelberg 2005;107-137.

Carr J, Macdonald DA, Waterhouse N. The blood supply

28. of the osteocutaneous free fibular graft. J Bone Joint Surg 1988; 70-B: 319-21.

Babak JM, Cordeiro PG. Avoiding and dealing with
29. Jong HL, Myeong JK, Jong WK. Mandibular reconstruction with free vascularized fibular flap. Journal

30. $1995 ; 23: 2-26$.

Abdel WAA. Osteocutaneous free fibular flap for anatomical reconstruction of the mandible following tumor resection.

31. Journal of the Egyptian Nat Cancer Inst 2003; 15(3): 243-51.

Joel F, Benoit P, Blandine R, Jacques M. Advantages and 32. Limitations of the Fibula Free Flap in Mandibular 55: 440-8.

Hidalgo DA, Pusic AL. Free-Flap Mandibular

Reconstruction: A 10-Year Follow-Up Study.Plastic and 438-49. Reconstructive Surgery 2002; 110(2): 